Original Article

\title{
Prior swimming exercise favors muscle recovery in adult female rats after joint immobilization
}

\author{
Ana Claudia Petrini ${ }^{1)}$, Douglas Massoni Ramos ${ }^{2)}$, Luana Gomes de Oliveira ${ }^{2)}$, \\ Carlos Alberto da Silva ${ }^{1,2)}$, Adriana Pertille ${ }^{1,2)^{*}}$ \\ 1) Graduate Program in Physiotherapy, Methodist University of Piracicaba, UNIMEP, Brazil \\ 2) Laboratory of Neuromuscular Plasticity, Graduate Program in Science of Human Movement, \\ Methodist University of Piracicaba, UNIMEP: Rodovia do Açúcar, 7000, CEP 13400-911, \\ Piracicaba, SP, Brazil
}

\begin{abstract}
Purpose] To evaluate the efficacy of pre-exercise on immobilization and subsequent recovery of white gastrocnemius (WG) and soleus (SOL) muscles of female rats. [Subjects and Methods] Thirty, 8-month-old, female Wistar rats were randomly and evenly allocated to six groups: sedentary (S); immobilized sedentary (IS); immobilized/rehabilitated sedentary (IRS); trained (T); immobilized trained (IT); and immobilized/rehabilitated trained (IRT). For four months, T, IT and IRT group animals performed swimming exercise (three sessions per week, 60 minutes per session), while S, IS and IRS groups animals remained housed in cages. After this period, the left hindlimb of the animals from the IS, IRS, IT and IRT groups was immobilized for five days, with the ankle at $90^{\circ}$. After removal of the orthosis, animals from the IRS and IRT groups followed a rehabilitation program based on swimming (five sessions per week, 60 minutes per session) for two weeks. [Results] Immobilization significantly reduced the cross-sectional area of the white gastrocnemius muscle; no changes were observed in the soleus muscles of the trained animals. Transforming growth factor- $\beta 1$ protein levels were similar among the trained groups. [Conclusion] Prior swimming prevents hypotrophy of the soleus muscle after immobilization, and protein levels reflected the adaptive capacity of the skeletal muscle.

Key words: Skeletal muscle, Immobilization, Exercise
\end{abstract}

(This article was submitted Feb. 11, 2016, and was accepted Apr. 7, 2016)

\section{INTRODUCTION}

Metabolic and functional changes that derive from the natural aging process increase the number of diseases, fractures, and falls experienced by individuals, thereby causing patients to experience brief periods of inactivity, which culminate in joint immobilization and may directly influence the development of age-related sarcopenia ${ }^{1)}$. Studies show that after 48 hours of joint immobilization, skeletal muscle develops atrophy ${ }^{2,3)}$, and that six hours after immobilization, a $37 \%$ reduction of protein synthesis occurs ${ }^{3,4)}$.

Upon joint immobilization, type I fibers (slow) are more vulnerable to hypotrophy due to their oxidative metabolic profile and to the anti-gravitational characteristics of postural or tonic muscles ${ }^{5,6}$.

Physical activity is potentially capable of minimizing the negative effects of aging ${ }^{7)}$ and immobilization ${ }^{8)}$. Prior strength training induces hypertrophy and protects the structural and mechanical properties of skeletal muscle during a disuse period, through the preservation of new cell nuclei that are developed in the muscle tissue during the training period ${ }^{9)}$. Therefore, the previous physical training load may influence muscle recovery after a period of inactivity ${ }^{10)}$. Aerobic training performed regularly favors the skeletal muscle cellular response through an improvement in capillary density ${ }^{11)}$.

High-intensity resistance training is an effective method for the treatment of atrophic muscles ${ }^{12}$. However, the effect of

*Corresponding author. Adriana Pertille (E-mail: apertille@unimep.br)

(C2016 The Society of Physical Therapy Science. Published by IPEC Inc.

This is an open-access article distributed under the terms of the Creative Commons Attribution Non-Commercial No Derivatives (by-nc-nd) License $<$ http://creativecommons.org/licenses/by-nc-nd/4.0/>. 
regular exercise without load on atrophy also needs to be investigated.

Based on the negative effects of joint immobilization on the aging process and on the cellular alterations derived from regular aerobic training, the hypothesis tested in the present study was that, training performed during a long period prior to immobilization might minimize the effects of muscle disuse and favor post-immobilization muscle recovery. Thus, the objective of this study was to evaluate the efficacy of pre-exercise on immobilization and subsequent recovery of the white gastrocnemius (WG) and soleus (SOL) muscles of female rats.

\section{SUBJECTS AND METHODS}

Thirty, 8-month-old, female Wistar rats were maintained under controlled temperature conditions, with 12-hour light/dark cycles, and with ad libitum access to food and water. This study was approved by the Ethics Commission for Animal Use (Comissão de Ética sobre o Uso de Animais -CEUA) of the Methodist University of Piracicaba under the protocol 09/13.

Female Wistar rats were randomly and evenly allocated to six groups: sedentary (S); immobilized sedentary (IS); immobilized/rehabilitated sedentary (IRS); trained (T); immobilized trained (IT); and immobilized/rehabilitated trained (IRT).

For four months, the T, IT and IRT group animals performed swimming exercise (water temperature $30{ }^{\circ} \mathrm{C} \pm 2{ }^{\circ} \mathrm{C}$ ) that lasted for 60 minutes per session, at a frequency of three sessions per week on alternate days, for a total of 48 training sessions. During this time, the S, IS and IRS group animals were housed in cages and could move freely.

After four months of training, the left hindlimbs of the animals in the IS, IRS, IT and IRT groups were immobilized for five days using an acrylic resin orthosis that kept the ankle at $90^{\circ}$ and the left knee and hip free, following the method described by Silva et $\mathrm{al}^{13)}$. Female rats from the IRS and IRT groups were physically rehabilitated in swimming sessions starting 24 hours after orthosis removal. Remobilization was performed five times per week (60-minute sessions without a load increase) for two weeks, a total of 10 sessions, according to the protocol described by Nascimento et al ${ }^{10)}$. All of the animals that performed swimming exercise had previously been adapted to this training. Animals in the T group continued the preimmobilization exercise protocol, as follows: three sessions per week, 60 minutes per session, for an additional six sessions.

The choice of females was based on the studies of Nascimento et al. ${ }^{10)}$ and Milani et al. ${ }^{14}$, which conducted swimming after training immobilization of female Wistar rats.

The animals were euthanized using $1.16 \mathrm{~g} / 10 \mathrm{ml}$ ketamine hydrochloride and $2 \mathrm{~g} / 100 \mathrm{ml}$ xylazine hydrochloride at a dose of $0.09 \mathrm{ml} / 100 \mathrm{~g}$ body weight. The left WG and SOL muscles were collected, weighed, and divided into two equal parts: one part was used to perform light microscopy, and the other part was used to perform immunoblotting.

Samples were fixed on wooden supports with tragacanth gum and were then frozen in $-80{ }^{\circ} \mathrm{C}$ isopentane and $-196{ }^{\circ} \mathrm{C}$ liquid nitrogen. The muscles were cross-sectioned on a cryostat (Microm-HS505E) to a thickness of $8 \mu \mathrm{m}$ and mounted on slides. The sections were then stained with hematoxylin and eosin (HE). To analyze the muscle cross-sectional area (CSA), 250 muscle fibers from each muscle per animal were assessed. The samples were viewed under a light microscope (20 $\times$ magnification) coupled to a camera and analyzed using Image Pro-Plus ${ }^{\circledR} 6.0$ software (Media Cybernetics).

The transforming growth factor (TGF)- $\beta 1$ protein level (TGF- $\beta 1$; mouse monoclonal antibody, Sigma-Aldrich, T7039, St. Louis, MO, USA) was quantified in all of the experimental groups. The muscles were homogenized in a buffer solution containing protease and phosphatase inhibitors. Muscle extracts were centrifuged for 20 minutes at $4{ }^{\circ} \mathrm{C}$; the supernatant was treated with Laemmli buffer (10\% SDS, 50\% glycerol, $0.1 \%$ bromophenol blue, $100 \mathrm{mM}$ Tris-HCl, $\mathrm{pH} 7.4)$, and $50 \mathrm{mg}$ of protein was loaded onto a $12 \%$ SDS-polyacrylamide gel. The proteins were transferred from the gel to a nitrocellulose membrane using electrophoretic transfer equipment from Bio-Rad. The membranes were blocked for one hour at room temperature (RT) in 5\% skim milk diluted in basal solution ( $10 \mathrm{mM}$ Trisma base, $150 \mathrm{mM} \mathrm{NaCl}$, and $0.02 \%$ Tween-20). Then, the membranes were incubated in the primary antibody overnight at $4{ }^{\circ} \mathrm{C}$, washed in basal solution, and incubated for 2 hours at RT in basal solution containing a peroxidase-conjugated secondary antibody (goat anti-mouse IgG-HRP, Santa Cruz: sc-2005, Dallas, TX, USA). To detect immunoreactive bands, the membranes were exposed using a chemiluminescence kit (Super Signal West Pico Chemiluminescence, Pierce, Therm Scientific, Waltham, MA, USA) for 5 minutes, and the signal was detected using G-Box equipment (Gene Sys).

The membranes were then washed in basal solution and incubated for one hour at $60^{\circ} \mathrm{C}$ in $10 \mathrm{ml}$ of stripping buffer (10 mM Tris- $\mathrm{HCl} \mathrm{pH} 7.5,0.1 \mathrm{M} \beta$-mercaptoethanol, $8 \mathrm{M}$ urea). Then, the membranes were incubated in $1 \mathrm{M} \mathrm{Tris-HCl}(\mathrm{pH}$ 7.5) for 30 minutes, to neutralize the stripping buffer, washed in basal solution, and processed as previously described to detect glyceraldehyde-3-phosphate dehydrogenase (GADPH mouse monoclonal, Santa Cruz, sc-59540, Dallas, TX, USA), which is an internal control protein, as its expression is not altered by changes in physiologic conditions. Optical densitometry quantification was performed using Image J software (The National Institute of Health, USA).

Statistical analysis was performed using BioStat version 5.9 software. The Shapiro-Wilk test was conducted to assess data normality. To compare the data of three groups, one-way ANOVA followed by Tukey's post-test was performed on the data with a parametric distribution, while the Kruskal-Wallis test was performed on the data with a non-parametric distribution. To perform a comparative analysis of two groups, Student's t-test for independent samples was used for parametric data, and the Wilcoxon test was used for non-parametric data. In all cases, statistical significance was accepted for values of $p<0.05$. 
Table 1. White gastrocnemius (WG) muscle and soleus (SOL) muscle mean weights in grams (g) and muscle fiber mean cross-section area $\left(\mu \mathrm{m}^{2}\right)$ with the standard deviation for all groups

\begin{tabular}{lcccc}
\hline Groups & \multicolumn{2}{c}{ Muscle weight $(\mathrm{g})$} & \multicolumn{2}{c}{$\mathrm{CSA}\left(\mu \mathrm{m}^{2}\right)$} \\
\cline { 2 - 5 } & WB & SOL & WB & SOL \\
\hline S & $0.417 \pm 0.07$ & $0.135 \pm 0.02^{*}$ & $3,884 \pm 510^{* *}$ & $3,446 \pm 356^{* *}$ \\
IS & $0.412 \pm 0.01$ & $0.132 \pm 0.03$ & $2,720 \pm 344$ & $2,234 \pm 91$ \\
IRS & $0.363 \pm 0.03$ & $0.121 \pm 0.02$ & $2,773 \pm 606$ & $2,636 \pm 315$ \\
T & $0.459 \pm 0.28$ & $0.172 \pm 0.01$ & $5,038 \pm 70 \dagger^{\dagger}$ & $3,926 \pm 310$ \\
IT & $0.426 \pm 0.11$ & $0.157 \pm 0.03$ & $3,515 \pm 560$ & $3,064 \pm 302$ \\
IRT & $0.381 \pm 0.06$ & $0.148 \pm 0.03$ & $3,043 \pm 505$ & $3,441 \pm 300^{\#}$ \\
\hline
\end{tabular}

S: sedentary; IS: immobilized sedentary; IRS: immobilized/rehabilitated sedentary; T: trained; IT: immobilized trained; IRT: immobilized/rehabilitated trained.

$*$ different from group T, $\mathrm{p}<0.01 ; * *$ different from groups IS and IRS, $\mathrm{p}<0.05 ;{ }^{\dagger}$ different from groups IT and IRT, p $<0.05$; ${ }^{*}$ different from group IRS, $\mathrm{p}<0.05$.

\section{RESULTS}

Training favored the gain of SOL muscle weight in T group animals compared with S group animals $(\mathrm{p}<0.01$, Table 1). Immobilization significantly reduced the WG CSA in the animals from the IS, IRS, IT, and IRS groups compared with the animals from the control groups ( $\mathrm{S}$ and $\mathrm{T}, \mathrm{p}<0.05$ ). Immobilization also significantly reduced the SOL CSA in the animals from the IS and IRS groups compared with the animals from the $S$ group $(p<0.05)$; however, no significant difference was found among the trained groups, and the IRT group showed a significant increase in CSA compared with the IRS group $(\mathrm{p}<0.05$, Table 1$)$.

Representative morphological effects of pre-exercise on immobilization are shown in Fig. 1. Note the atrophy of the muscle fibers of the immobilized animals (Fig. 1B, C, H, I) and increased CSA of the soleus muscle fibers in the IRT group (Fig. 1L).

The level of TGF- $\beta 1$ protein in the WG muscle was higher in the $\mathrm{S}$ group than in the IS, IRS, and T groups ( $<<0.05$ ), but no significant difference was found among animals from the trained groups. Immobilization induced an increase in SOL TGF- $\beta 1$ in the IS group compared with that in the other sedentary groups $(p<0.05)$. SOL TGF- $\beta 1$ was similar among the trained groups, with TGF- $\beta 1$ in the IRT group being significantly higher than that of the IRS group (p<0.02, Fig. 2).

\section{DISCUSSION}

Pre-immobilization training was able to prevent hypotrophy of the SOL muscle (mainly composed of type I fibers), as immobilization did not reduce the CSA in the IT group, and during the rehabilitation period, the CSA was higher in the IRT group than in the IRS group. The WG muscle (mainly composed of type II fibers) exhibited CSA reduction after immobilization in both the IS and IT groups compared with their respective controls, the S and T groups, respectively.

A short immobilization period, ranging from four to ten days, is sufficient to cause metabolic and structural changes to muscle tissue $2,13,15,16$ ). The results of the present study show that five days of inactivity, even with an orthosis model that allowed for unloading of the animal, were sufficient to induce changes in the muscles analyzed, particularly in the SOL. Changes in the joint, such as adhesions of fibrous hyperplasia tissue in the synovial membrane and fibrosis in the articular cartilage surface, are also observed in the early hours of immobilization ${ }^{17}$.

These data underline the clinical importance of having a rehabilitation program for patients who undergo joint immobilization, even for short periods of time. In addition, it is noteworthy that according to Wall, Dirks, and Van Loon ${ }^{18)}$, the repetitive exposure of individuals to short periods of inactivity might culminate in the development of sarcopenia. Pitanga et al. ${ }^{19)}$ emphasize that reduced levels of physical activity also contribute to the development of sarcopenia and that regular physical exercise is a protective factor against sarcopenia in women in the postmenopausal period.

The pre-immobilization, long-term training protocol chosen for use in the present study was an attempt to approximate the protocols used in humans and was performed three times per week, without a load, for a total of 180 minutes every week. It was based on the World Health Organization recommendations for adults ${ }^{20)}$. The remobilization protocol considered that striated skeletal muscles require more time to recover than to undergo atrophy ${ }^{8,14)}$.

Although no significant differences were observed between the CSA of sedentary and trained female rats, the SOL muscle weight was significantly higher in the trained female rats than in the sedentary animals.

The results show that swimming remobilization did not contribute to the recovery of WG muscle CSA, which can be justified by the physical training performed. Milani et al. ${ }^{14)}$ compared the biomechanical effects of immobilization for seven days and remobilization in the skeletal muscle of trained and sedentary female rats. Training was conducted with swimming 


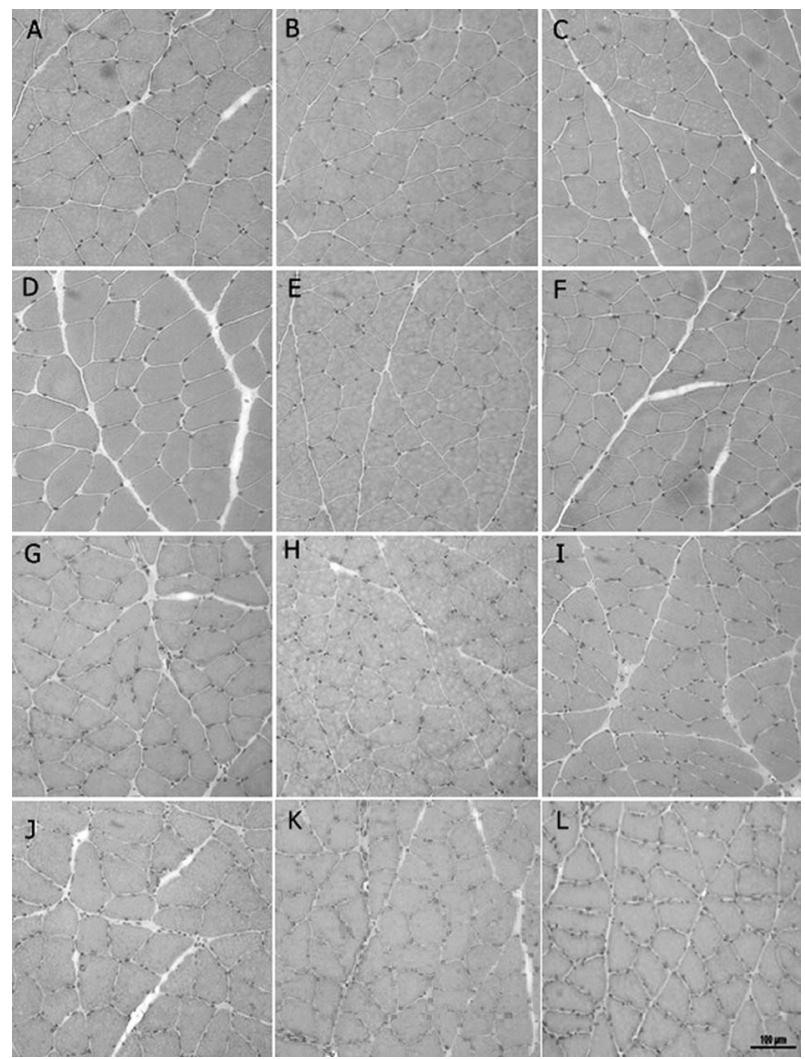

Fig. 1. Cross sections of white gastrocnemius (A-F) and soleus (G-L) muscle stained with HE

A and G: sedentary (S); B and H: immobilized sedentary (IS); C and I: immobilized/rehabilitated sedentary (IRS); D and J: trained (T); E and K: immobilized trained (IT); F and L: immobilized/rehabilitated trained (IRT). Observe reduction in cross-sectional area of the muscle fibers of immobilized groups $(\mathrm{B}, \mathrm{C}, \mathrm{H}, \mathrm{I})$. Bar $=100$ micron
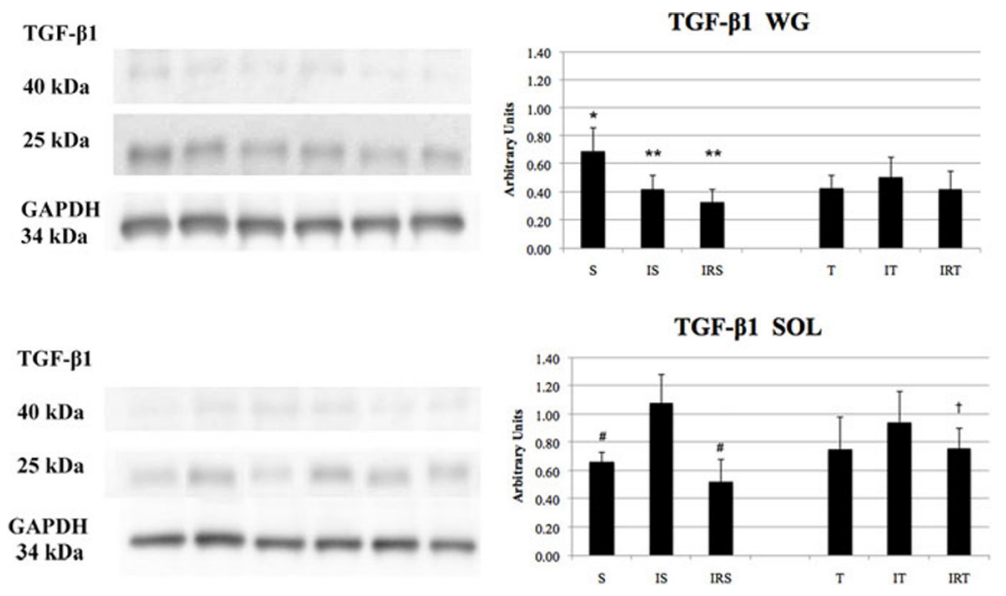

Fig. 2. White gastrocnemius (WG) muscle and soleus (SOL) muscle TGF- $\beta 1$ mean protein levels in arbitrary units, with the standard deviation

S: sedentary; IS: immobilized sedentary; IRS: immobilized/rehabilitated sedentary; T: trained; IT: immobilized trained; IRT: immobilized/rehabilitated trained. * different from group T; ** different from group S; \# different from group IS; † different from group IRS; p0.02

(60-minute sessions, five times per week) with a progressive load increase (up to $8 \%$ of body mass) for six weeks. Milani et al. ${ }^{14)}$ observed that compared with the sedentary group, the group that underwent pre-immobilization training presented a positive effect on the mechanical behavior of the gastrocnemius muscle after a two-week remobilization swimming plan, 
without a load increase.

Notably, coupling an external weight equivalent to $6 \%$ of the body mass in experimental models during swimming training is considered high-intensity physical activity ${ }^{10,21)}$.

Tanaka et al. ${ }^{22}$ observed that the soleus (predominantly type I fibers) and plantaris muscles (predominantly type II fibers) are able to recover after 14 days of tail suspension and three days of reloading, however, the process leading to recovery seems to differ. The production of hepatocyte growth factor (HGF), a mitogen for satellite cells, in the soleus muscle was approximately 3 -fold greater than that of controls.

The TGF- $\beta 1$ cytokine family is known to regulate several cellular processes, such as angiogenesis, differentiation, and apoptosis, in addition to stimulating fibroblast proliferation and extracellular matrix production ${ }^{23}$. Following a muscle lesion, TGF- $\beta 1$ increases, which may inhibit regeneration and promote fibrosis ${ }^{24)}$.

Czarkowska-Paczek et al. ${ }^{25}$ ) investigated the effects of training on the formation of TGF- $\beta 1$ in the SOL muscle and observed increased mRNA expression but reduced protein levels after six weeks of treadmill training. Because TGF- $\beta 1$ is a strong stimulator of collagen expression, the increase of TGF- $\beta 1$ in muscle might be harmful, and on the basis of the cellular responses to training, Czarkowska-Paczek et al. ${ }^{25}$ ) suggested that this reduction might be due to a regulatory mechanism of transcription.

The results of the present study show there was a similar level of TGF- $\beta 1$ among the trained groups, in both muscles, which suggests that a trained muscle has the capacity to adapt to different stimuli, such as immobilization and remobilization. In groups of sedentary animals, however, immobilization increased the level of TGF- $\beta 1$ in the WG and SOL muscles only in the IS group.

Future studies are necessary to compare aerobic and anaerobic training with a similar weekly frequency, and to perform additional molecular analyses, such as the quantification of TNF- $\alpha$, metalloproteinases, free radical markers, collagen and satellite cells, as well as functional tests.

In conclusion, pre-exercise prevents hypotrophy of the soleus muscle after immobilization. The TGF- $\beta 1$ protein level highlights the muscle's adaptive capacity, indicating that regular exercise, even without loading and performed three times per week, can have positive effects on the skeletal muscle of female rats.

\section{REFERENCES}

1) Wall BT, van Loon LJ: Nutritional strategies to attenuate muscle disuse atrophy. Nutr Rev, 2013, 71: 195-208. [Medline] [CrossRef]

2) Carvalho LC, Polizello JC, Padula N, et al.: Propriedades mecânicas do gastrocnêmio eletro estimulado pós-imobilização. Acta Ortop Bras, 2009 , 17: 269-272. [CrossRef]

3) Kodama FY, Camargo RC, Job AE, et al.: Muscle mechanical properties of adult and older rats submitted to exercise after immobilization. Acta Ortop Bras, 2012, 20: 218-222. [Medline] [CrossRef]

4) Williams PE, Catanese T, Lucey EG, et al.: The importance of stretch and contractile activity in the prevention of connective tissue accumulation in muscle. J Anat, 1988, 158: 109-114. [Medline]

5) Lieber RL: Skeletal muscle structure, function \& plasticity: The physiological basis of rehabilitation, 2nd ed. Baltimore: Williams \& Wilkins, 2002, pp $183-226$.

6) Minamoto BV: Classificação e adaptações das fibras musculares: uma revisão. Fisioter Pesqui, 2005, 12: 50-55.

7) Degens H: Age-related skeletal muscle dysfunction: causes and mechanisms. J Musculoskelet Neuronal Interact, 2007, 7: 246-252. [Medline]

8) Kannus PL, Jozsa M, Restro M, et al.: The effects of training, immobilization and remobilization on musculoskeletal tissues. 1.Training and immobilization. Scand J Med Sci Sports, 1992, 2: 100-118. [CrossRef]

9) Bruusgaard JC, Johansen IB, Egner IM, et al.: Myonuclei acquired by overload exercise precede hypertrophy and are not lost on detraining. Proc Natl Acad Sci USA, 2010, 107: 15111-15116. [Medline] [CrossRef]

10) Nascimento CC, Padula N, Milani JG, et al.: Histomorphometric analysis of the response of rat skeletal muscle to swimming, immobilization and rehabilitation. Braz J Med Biol Res, 2008, 41: 818-824. [Medline] [CrossRef]

11) Adolfsson $\mathrm{J}$ : The time dependence of training-induced increase in skeletal muscle capillarization and the spatial capillary to fibre relationship in normal and neovascularized skeletal muscle of rats. Acta Physiol Scand, 1986, 128: 259-266. [Medline] [CrossRef]

12) Tanaka S, Obatake T, Hoshino K, et al.: Influence of exercise intensity on atrophied quadriceps muscle in the rat. J Phys Ther Sci, 2015, 27: 3445-3450. [Medline] [CrossRef]

13) da Silva CA, Guirro RR, Polacow ML, et al.: Rat hindlimb joint immobilization with acrylic resin orthoses. Braz J Med Biol Res, 2006, 39: 979-985. [Medline] [CrossRef]

14) Oliveira Milani JG, Matheus JP, Gomide LB, et al.: Biomechanical effects of immobilization and rehabilitation on the skeletal muscle of trained and sedentary rats. Ann Biomed Eng, 2008, 36: 1641-1648. [Medline] [CrossRef]

15) Kannus P, Jozsa L, Järvinen TL, et al.: Free mobilization and low- to high-intensity exercise in immobilization-induced muscle atrophy. J Appl Physiol 1985, 1998, 84: 1418-1424. [Medline]

16) Durigan JL, Cancelliero KM, Dias CN, et al.: Efeitos da imobilização articular aguda nos músculos do membro posterior de ratos: análise metabólica e morfométrica. Fisiot Pesqui, 2006, 13: 38-45.

17) Kojima S, Hoso M, Watanabe M, et al.: Experimental joint immobilization and remobilization in the rats. J Phys Ther Sci, 2014, 26: 865-871. [Medline] [CrossRef] 
18) Wall BT, Dirks ML, van Loon LJ: Skeletal muscle atrophy during short-term disuse: implications for age-related sarcopenia. Ageing Res Rev, 2013, 12: 898-906. [Medline] [CrossRef]

19) Pitanga CP, Pitanga FJ, Gabriel RE, et al.: Association and discriminatory power of physical activity for the prevention of sarcopenia in postmenopausal women. Motricidade, 2015, 11: 114-122. [CrossRef]

20) World Health Organization: Global Recommendations on Physical Activity for Health. Geneva: WHO, 2011.

21) Peijie C, Zicai D, Haowen X, et al.: Effects of chronic and acute training on glucocorticoid receptors concentrations in rats. Life Sci, 2004, 75: 1303-1311. [Medline] [CrossRef]

22) Tanaka S, Tachino K, Kawahara E, et al.: Hepatocyte growth factor in mouse soleus muscle increases with reloading after unloading. J Phys Ther Sci, 2006, 18: 33-41. [CrossRef]

23) Heldin $\mathrm{CH}$, Miyazono K, ten Dijke P: TGF- $\beta$ signalling from cell membrane to nucleus through SMAD proteins. Nature, 1997, 390: 465-471. [Medline] [CrossRef]

24) Smith CA, Stauber F, Waters C, et al.: Transforming growth factor-beta following skeletal muscle strain injury in rats. J Appl Physiol 1985, 2007, 102: 755-761. [Medline] [CrossRef]

25) Czarkowska-Paczek B, Zendzian-Piotrowska M, Bartlomiejczyk I, et al.: The effect of acute and prolonged endurance exercise on transforming growth factor- $\beta 1$ generation in rat skeletal and heart muscle. J Physiol Pharmacol, 2009, 60: 157-162. [Medline] 